\title{
Tangence
}

\section{La machine humaine à l'épreuve de la vitesse : L'homme pressé , de Paul Morand}

\section{Robert Dion}

Numéro 55, septembre 1997

La vitesse

URI : https://id.erudit.org/iderudit/025949ar

DOI : https://doi.org/10.7202/025949ar

Aller au sommaire du numéro

Éditeur(s)

Tangence

ISSN

0226-9554 (imprimé)

1710-0305 (numérique)

Découvrir la revue

Citer cet article

Dion, R. (1997). La machine humaine à l'épreuve de la vitesse : L'homme pressé de Paul Morand. Tangence, (55), 95-107. https://doi.org/10.7202/025949ar d'utilisation que vous pouvez consulter en ligne.

https://apropos.erudit.org/fr/usagers/politique-dutilisation/ 


\section{La machine humaine à l'épreuve de la vitesse : L'homme pressé, de Paul Morand}

\section{Robert Dion}

Le monde est biffé par la promptitude.

Pierre Drieu la Rochelle, "Auto", Fond de cantine, 1920

L'art de Paul Morand (1888-1976), écrivain qu'on ne lit plus guère aujourd'hui, est un art du contretemps. Morand, en effet, n'a jamais été tout à fait de son temps, simplement en avance sur lui ou en dehors, à côté, prolongeant indéfiniment l'écho des roaring twenties, jusque dans la France giscardienne. Jacques Laurent (alias Cécil Saint-Laurent), l'un des "hussards " des années 1950 qui s'étaient reconnus en Morand, disait de celui-ci qu'il regrettait l'Europe d'avant 1914 et en même temps était avide de toute nouveauté, qu'il ne faisait en somme "aucune différence entre la mode et l'éternité " ${ }^{1}$. Comme Stefan Zweig, son aîné de sept ans, l'écrivain français serait resté attaché au "monde d'hier " ${ }^{2}$, à la civilisation européenne anéantie avec la Première Guerre mondiale, nostalgie qu'il aurait cependant, à la différence de l'Autrichien, surmontée, se commettant à plusieurs titres avec le régime de Vichy, alors que Zweig fuira devant Hitler puis se donnera la mort en 1942, au Brésil.

Morand, lui, aura survécu à la perte d'un monde d'insouciance et de culture ${ }^{3}$, de cosmopolitisme, de luxe et de voyages - celui dont il est question, par exemple, dans le diptyque Ouvert la nuit et Fermé la nuit ${ }^{4}$. Il aura misé sur la vitesse, pris

1 Cité dans louvrage de Jean-François Fogel, Morand-Express, Paris, Grasset, 1980, p. 55.

2 Du titre de l'uautobiographie d'une génération * de Zweig, qui est peut-être son plus beau livre, Die Welt von Gestern, Stockholm, Bermann-Fischer Verlag, 1944.

3 Zweig parle, dans l'incipit de son autobiographie, du agoldene Zeitalter der Sicherheit * - de l'âge d'or de la sécurité.

4 Ces deux recueils de nouvelles, parus respectivement en 1922 et en 1923 , ont été repris dans le tome I des Nouvelles complètes de Paul Morand, Paris, 
96

de vitesse sa nostalgie, même s'il s'attarde parfois complaisamment à décrire le crépuscule européen. Dans sa volonté de vivre vite, il aura pleinement participé au mouvement de l'entre-deuxguerres, à cette ivresse d'oubli forcené, de plaisir inquiet. L' impermanence" aura été son destin, sa manière d'échapper à la cristallisation du souvenir, qui du passé tire de toujours nouvelles perfections.

Avec Morand-Express, Jean-François Fogel a signé un beau livre sur le "possédé du mouvement " qu'a été l'auteur d'Hécate et ses chiens; inutile, donc, d'y revenir plus longuement. Il serait tout aussi vain de vouloir situer Morand parmi les écrivains de son temps: vaguement surréaliste à ses débuts, dans les quelques plaquettes de poèmes qu'il a publiées entre 1919 et 1928 (la première porte le titre, éloquent, de Lampes à arc, la dernière, d'U.S.A. 1927), il trouve bientôt sa voie (rapide?) et ne cultive par la suite que des amitiés littéraires (Giraudoux, Chardonne, etc.) sans incidence réelle sur son œuvre. L'écrivain de droite, à ce qu'il semble, est un chasseur solitaire - et, du reste, qui veut voyager vite ne s'encombre ni de collègues ni de disciples; pour Morand, cela viendra plus tard, dans les années 1950, alors que, isolé par ses choix politiques des années de guerre, il fera bon accueil à la ferveur des "hussards".

Dans le cadre du présent dossier consacré à la vitesse, je voudrais m'arrêter au roman de Morand paru en 1941, L'bomme pressé $^{-5}$. Déjà le titre, que les critiques ont d'emblée associé à l'auteur lui-même, forme une catachrèse qui désigne tout le personnage - dont c'est l'unique qualité : Jean Richer parle d'un "monomane de la vitesse" 6 - et toute l'action: aller presto, faire tout "vite et mal. (p. 45). La vitesse est l'objet central d'un roman qui, comme l'a remarqué Richer, tient du récit allégorique, de la "moralité ${ }^{7}$, ce qui ne facilite pas les choses, tant s'en faut: le

Gallimard, 1992, coll. “Bibliothèque de la Pléiade " (édition présentée, établie et annotée par Michel Collomb).

5 L'bomme pressé, Paris, Gallimard, 1941. Je me référerai à l'édition de poche parue dans la collection "Folio * en 1972; les références seront désormais indiquées par le seul folio entre parenthèses.

6 Jean Richer, "L'obsession de la vitesse comme Erinye : L'bomme pressé aujourd'huì, Corps écrit, $\mathrm{n}^{\circ} 24$, décembre 1987, p. 89-92. Il est à noter que cette livraison de Corps écrit est entièrement consacrée au thème de la vitesse.

7 Ibid., p. 89. 
discours sur la vitesse est si développé, sa mise en scène si caricaturale, qu'on échappe difficilement au redoublement, à la redite; de plus, la critique de la vitesse - d'une vitesse néanmoins fascinante, authentiquement moderne - est si visible, si outrée, qu'elle s'assimile à une charge parodique, à une condamnation sans appel de toute précipitation. Pourtant, l'homme pressé, ce Pierre Nioxe tellement aérien (ne retrouve-t-on pas dans son patronyme les premières lettres d'oxygène?), ce "vélociférique" comme l'appelle le docteur Regencrantz reprenant le mot de Goethe, ne représente-t-il pas aussi une figure emblématique des changements culturels qu'allaient causer les technologies de la vitesse dans la seconde partie du $\mathrm{xx}^{\mathrm{e}}$ siècle? Homme du "Temps des Machines" (qui est celui de la thermodynamique), pour dire comme Jacques Attali ${ }^{8}$, Nioxe serait également celui d'un temps propre, relatif (au sens d'Einstein), qui n'est pas le temps biologique (on verra que ce temps joue contre lui), mais un temps à sa mesure, qui, sous ce rapport, peut faire penser à celui du petit Oskar dans Le tambour de Günter Grass ${ }^{9}$; toutefois, alors qu'Oskar décide de cesser de grandir et d'opposer au rythme du temps nazi un temps arrêté, l'homme pressé choisit d'accélérer son propre temps, au risque de crever les machines (au début du récit, il percute avec sa voiture un camion immobilisé) ou de se crever lui-même (ce qui se produit à la fin).

Fogel remarque à juste titre que L'bomme pressé vient à contretemps: en 1941, alors que la France est littéralement asphyxiée, que le carburant manque et que les déplacements se compliquent, Morand écrit l'histoire d'un agité qui se moque de l'espace et des distances, le désignant tel un "mangeur d'espace" (p. 135); "Telrreur sociologique", conclut Fogel ${ }^{10}$. À la décharge de l'auteur, soulignons que l'action du roman, quoique non datée, se déroule vraisemblablement dans l'entre-deux-guerres, avant le début du second conflit mondial, donc. Déphasée par rapport au temps de l'écriture, l'action, en revanche, rend bien compte des décennies 1920 et 1930 , et notamment de l'intérêt alors porté à la vitesse mécanique personnalisée, intérêt nouveau à cette époque $s^{\prime}$ il faut en croire Christophe Studeny ${ }^{11}$. Par

8 Jacques Attali, Histoires du temps, Paris, Fayard, 1982.

9) l'exemple est d'Attali, ibid., p. 311.

10) Fogel, op. cit., p. 230.

11 Christophe Studeny, Linvention de la vitesse. France, $x w{ }^{\circ}-x x^{*}$ siecle, Paris. Gallimard, 1995, coll. "Bibliothèques des histoires", p. 291. 
rapport aux romans du chemin de fer et, partant, de la vitesse mécanique collective, dont le prototype est La bête bumaine de Zola (1890), L'bomme pressé représente sans contredit un roman de l'automobile personnelle et de l'avion, bien que le métro et le paquebot transatlantique y soient aussi utilisés. Il s'agit là, on en conviendra, d'une donnée capitale pour l'analyse.

\section{La vitesse et ses ratés}

Lancé à "un train d'enfer" - c'est le titre de la première partie du roman -, le héros connaît bientôt "le prix du temps" intitulé de la seconde partie. De manière significative, en une sorte de mise en abyme, le protagoniste de L'homme pressé apparaît dès la première phrase du livre dans une situation de rupture par rapport au mouvement général (dans les deux sens du terme: locomotion et courant social): "Au moment où la route atteignait le sommet de la butte et allait redescendre l'autre versant, l'homme sauta du taxi sans attendre le coup de frein du chauffeur" (p. 11). Pierre Nioxe est précisément celui qui saute en marche - alors que la route redescend, qu'elle abandonne le sommet, la crête; il n'attend pas d'être parvenu à destination, il s'enfuit à mi-trajet, préfiguration de sa mort à trente-cinq ans, au beau milieu de la vie. Cette précipitation du héros lui fait devancer les échéances, précéder l'accomplissement naturel des choses, bref, bousculer ce qui ne peut l'être: d'où le tragique du personnage - et son caractère décalé.

Car Morand n'a pas fait de Pierre Nioxe une créature de génie, tout au plus un possesseur du "don fatal [...] de la mobilité"; les voitures, les avions ne constituent que des prothèses, c'est-à-dire les moyens de sa frénésie. Or si cette frénésie permet à Pierre de réussir de bons coups - dans son domaine, l'antiquité, "il faut faire vite [...] comme dans le reste" (p. 17) -, elle lui fait le plus souvent tout rater: ses affaires, ses plaisirs (Nioxe ne supporte pas le rituel des repas, non plus que celui du théâtre), son mariage. Là réside sans doute la critique de la vitesse la plus diégétiquement fondée. Nioxe est désigné par le narrateur comme un "homme déréglé" (p. 121), et il se décrit luimême comme un être désaccordé (p. 17). Tout le récit est construit sur cette idée: son principal ressort est l'exposition, voire la théorisation, du fondamental désaccord entre le héros et le monde. 
"Je pense que la vitesse est la forme moderne de la pesanteur et je sais que j'obéis à l'impulsion vraie de l'univers et je suis le seul à sentir que j’y obéis"(p. 18): telle est, d'entrée de jeu, la profession de foi clu protagoniste. Ce sentiment euphorique d'accord avec le temps physique (celui de la chute des corps, indexé ici par la "pesanteur) ne saurait hélas durer, puisque les lois physiques, qui, dans la chronométrie classique, renvoient le temps à l'espace par un mouvement approprié, entrent en concurrence avec d'autres temps, avec d'autres aperceptions du temps vécul. Au temps physique ${ }^{12}$ se superposent en effet un temps cosmologique, un temps biologique, un temps psychologique, et ainsi de suite: l'être humain se meut à l'intérieur de ces divers temps, dont il s'accommode plus ou moins aisément. Bergson distingue pour sa part un temps quantitatif, celui des chronomètres, et un temps qualitatif, qui se réfère à la durée intérieure. Bref, pressé ou non, l'homme ne saurait vivre dans le seul temps physique, celui de la distance-temps, comme le désire Pierre Nioxe; il est au contraire plongé dans un temps stratifié, qu'on appellera, pour le clistinguer du temps physique, temporalité, ou temps vécu par la conscience, et dont la stratification même lui fait ordinairement perdre la notion du temps.

Ainsi, Pierre Nioxe veut vivre dans le temps pur, alors que l'humanité vit une temporalité variable selon les époques et les cultures. Si la montre, instrument de la mesure du temps, est son amie (p. 11), la nature, et le temps cosmologique correspondant, lui est hostile; opposée au monde de la vitesse rationalisée, celleci, note Studeny, reste lente, imprévisible, et devient un facteur de discordance ${ }^{13}$. L'une des premières perceptions de Nioxe dans le roman est au demeurant celle de l'astre solaire qui, "pareil à un acteur qui ne peut se résoudre à quitter la scène, [...] se trânait dans le crépuscule d'été de neuf heures" (p. 11-12). Le personnage pousse la fébrilité jusqu'à nier tout cycle naturel, s'attaquant ainsi aux temps cosmologique et biologique; il fait fi du repos nocturne (la scène de l'accident de voiture a lieu nuitamment, alors que Pierre roule à tombeau ouvert - p. 45-48), du rythme de croissance des plantes (l'un des moments les plus comiques

\footnotetext{
12 Il serait plus juste de parler de "temps physicues" au pluriel, puisque les scientificpues admettent une pluralité des temps, du fait de l'impossibilité de rapporter tous les phénomènes de l'univers à un seul temps.

Studeny, op cit., p. 337.
} 
100

du livre est celui où le héros tente d'accélérer la croissance des fleurs à coup de Subitose, de Précipital, d'Activitte, de Superroburelle et autres fantaisistes substances - p. 191-196) et, surtout, de la durée de la gestation humaine: la rupture avec sa femme Hedwige, parangon de lenteur et de nonchalance, qui comme la nature a l'éternité devant elle (en vertu de son nom même: Hedwige contient ewig, "éternel" en allemand), est consommée lorsque Pierre, ne pouvant se résoudre à attendre neuf mois la venue de leur enfant, lui demande après sept mois de recourir à une césarienne.

\section{Le corps-machine}

Nioxe est donc en butte au temps biologique. Il n'ignore pas que, parallèlement aux temps intérieur et extérieur, s'écoule un "temps organique qui est celui de notre corps tout occupé à vieillir, de notre corps qui sait, avec l'exactitude terrible de l'inconscient, combien de battements il lui reste à faire avant la tombe" ( p. 167). Or le battement, scansion du temps biologique, rythme également la mesure de l'heure et le mouvement alternatif des machines; en cela, il constitue l'une des images privilégiées du roman, un connecteur des isotopies du corps et de la machine; le battement du coeur devient à ce point obsédant, à la fin du récit, que Pierre doit faire un effort pour ne pas l'entendre. Tout au long du roman, le héros est fasciné par les battements organiques et mécaniques; à propos d'horloges aperçues en vitrine, il s'enthousiasme:

Que de minutes ont bien pu débiter ces cadrans depuis cent cinquante ans! [...] Pensez-y... quel ressort humain pourrait lutter contre les leurs? Quelles diastoles, quelle systoles égaleront jamais leurs palettes et leurs cliquets! (p. 142)

L'image du battement n'est pas la seule qui permette d'assimiler la machine humaine à une mécanique; il en va ainsi, par exemple, du tube: "[l]es tuyaux sont toujours trop étroits, que ce soit un tuyau de vidange, un tuyau d'arrivée d'eau ou un goulot de bouteille, un larynx humain ou le tube de l'osophage" (p. 27).

Selon Hedwige, Pierre n'est pas un être humain mais une locomotive (p. 246), une mécanique automotrice et dangereuse. Du moins est-ce ainsi qu'elle est peu à peu amenée à le considérer: "[m]ais l'image se dérobait; elle ne voyait plus qu'un graphique, 
qu'une épure de machine, un robot à cent bras coudés qui tournoyait dans la chambre, happant les objets, les broyant, la happant elle-même, la faisant tournoyer, la mettant en danger, elle et l'enfant" (p. 242). L'“homme électrique" (p. 140) ne peut pas toujours traverser les autres à la manière d'un rayon; il doit forcément se frotter à leur lenteur, perdant ainsi de sa précieuse énergie. Une collision violente n'est pas non plus à exclure, qui risque de procluire le choc de l'intimité: "quel choc, pense Pierre, le jour où nous nous rencontrerons! ce qui s'appelle se rencontrer! c'est-à-dire échanger constance, chaleur, vitalité, le tout dans une intimité que je n'ai jamais connue jusqu'ici" (p. 102). L'homme pressé se trouve en fait à jouer la vitesse contre l'intimité, autre stratégie moderne: aller vite, c'est en effet se dérober à la société des hommes (qui ne font alors que passer), se murer dans l'instant. Le narrateur ne note$\mathrm{t}$-il pas, à la fin du roman, que le protagoniste, qui meurt solitaire, "avait vécu trop vite pour être remarqué. (p. 274)? Ainsi, Nioxe ne part jamais, il s'éclipse; il ignore les transitions, ne pratique que la disparition. Il est, à proprement parler, l'a homme-instantané " (p. 61), l'uhomme-torpille" des futuristes italiens ${ }^{11}$.

Comme les automobiles et les avions, Nioxe a "la beauté de la vitesse", qui est d'ailleurs la principale que lui prête le roman; et il en a la force guerrière, agressive, reconnue par Marinetti et par l'ensemble des fascismes du début de ce siècle ${ }^{15}$. Lancé en voiture sur les routes, il ne s'arrête pas même pour faire le plein, pratiquant le ravitaillement en vol (p. 27) ainsi que le font les avions de guerre. Puisant à une autre des grandes sources de l'imaginaire de l'énergie, imaginaire largement sollicité par la droite européenne des années 1920 et 1930, Morand associe son personnage à un héros sportif; dans un moment de pleine communion au mythe du sport tel qu'il s'est exprimé dans les premières décennies du $\mathrm{xx}^{\mathrm{C}}$ siècle (évoquons seulement les Olympiades de 1936 à Berlin), Pierre Nioxe soliloque:

14 Voir à ce propos fanette Roche-pézard, "Autour de la forme brève: les "démentes écritures" de F. T. Marinetti", Études littéraires, vol. 19, $\mathrm{n}^{2} 2$, automne 1986, p. 23-34.

15 En ce qui concerne les liens entre vitesse et violence, voir l'ensemble des tratvaux de Paul Virilio, et spécialement La vitesse de libération, Paris, Galiléc, 1995. Voir aussi, sur Virilio, l'article de Rémy Paindavoine, "Vitesse et Disparition. La "dromologie" de Paul Virilio", Études, décembre 1994, p. 619-628, où il est notamment question de la vitesse comme création militaire, et de lá guerre en tant que "guerre du temps". 
102

Seul!... Au fond, c'est justice; qu'est-ce que la vitesse sinon une course gagnée dont la solitude est le prix. On sème ses semblables... On les sème dans l'espoir que l'espèce n'en repoussera plus, compléta Pierre rageusement. Moi, je suis le champion de l'épreuve; j'ai résisté mieux que les autres à cette pression invisible qui s'infiltre en nous et qui s'appelle la lenteur. Je suis un spectacle sportif, conclut-il orgueilleusement. (p. 101)

On notera ici la violence de la compétition sportive, qui vise à l'éradication totale de la lenteur et de ses incarnations. Le combat entre vitesse et lenteur est du reste sans quartier; à la suite du passage que je viens de citer, Pierre ajoute: "[l]e jour où la lenteur sera plus forte que moi, je mourrai asphyxié. La mort n'est probablement qu'une différence de pression entre le milieu extéricut et notre être intérieur; quand l'extétieur devient plus fort, on creve" (p. 101). Ailleurs, toujours en ce qui concene le lien entre vitesse (ou plutôt: rapiclité) et lenteur, entre vitesse et mont, k protagoniste répond à son bien nommé ami Placide, qui lui fait remarquer qu'il serait bien ennuyé si le monde allait son train et le rattrapait: "[c]e jour-là, depuis longtemps, je serai mort" (p. 45$)$.

Or le personnage meurt bel et bien à la fin, vaincu par la lenteur ou au contraire rattrapé par le monde - cela est difficile à dire. À lire de près L'bomme pressé, il semble que le temps gagné soit, en définitive, perdu: chacun disposerait d'une longueur donnée de "ruban de vie", et la durée de l'existence tiendrait à la vitesse de déroulement de ce ruban. Pierre aurait tout simplement déroulé le sien plus rapidement que les autres; bien qu'à sa mesure, le temps lui aurait été mesuré. Le précurseur, celui qui "Court devant" (p. 45), ne saurait somme toute se prévaloir que du privilège de finir sa course avant les autres et n'aurait pas le loisir de courir une plus longue distance. La conception romanesque du temps biologique, on le voit, débouche ici sur une moralité immédiatement décodable.

\section{La technologie et la mort}

À ce stade-ci de l'analyse, il convient de s'attarder un moment aux circonstances de la mort de Pierre Nioxe, laquelle, chez ce devancier en tout, survient en fait avant sá mort véritable (située de toute façon hors roman), dans un avion au-dessus de la ville 
de New York. De toute évidence, ce n'est pas un hasard si l'infarctus qui va terrasser le protagoniste se produit à bord d'un aétoplane survolant la métropole américaine. Morand a publié en 1930 un ouvrage consacré à New York ${ }^{16}$, un carnet de voyage qui constitue sans doute l'un des plus vibrants portraits qu'un écrivain français ait tracé de cette ville. Au fil d'une visite systématique de Manhattan, de Downtown jusqu'à Uptown, Morand a voulu témoigner de l'esprit des lieux; dans le chapitre de clôture, il commente en ces termes la violence et la vitesse de la cité (choses dont on a vu qu'elles étaient liées chez plusieurs écrivains d'avant 1940):

Champ de bataille.

Une confusion terrible règne, comme pendant l'assaut. Le roc tremble, le macadam frémit.

New York est grand, il est neuf, mais grande et neuve, toute l'Amérique l'est [...]. Ce que New York a de suprêmement beau, de vraiment unique, c'est sa violence. Elle l'ennoblit, elle l'excuse, elle fait oublier sa vulgarité. Car New York est vulgaire; il est plus fort, plus riche, plus neuf que n'importe quoi, mais il est commun. La violence de la ville est dans son rythme. ${ }^{17}$

Curieusement, après avoir été saisi par la frénésie de New York puis de Chicago, frénésie encore augmentée par les moyens technologiques ("J'aime le rythme des grands ganglions urbains, l'animation de la circulation et le bondissement de ces ascenseurs au haut des maisons droites comme des avenues; la conductibilité de toute cette matière américaine me met dans un état d'éréthisme bienfaisant" - p. 249), Nioxe est bientôt irrité par ce qu'il se met à percevoir comme une "apathie américaine" (p. 250). Désenchanté, il s'interroge: "[q]ui a jamais pensé, avant les Américains, à se reposer? Tout ici est prétexte à fêtes et à lambinage..." (p. 250). Deux explications à ce revirement: ou bien Nioxe est parvenu à une espèce d'au-delà de la vitesse (vitesse qui, cu coup, menace son intégrité corporelle même), ou bien il ne la voit plus parce qu'il se trouve pris dans son flux, ce qui montrerait bien que le temps de la vitesse est relatif ${ }^{1 r}$, n'est

16 P'at Morand, New York, Paris, Garnict-Flammarion, 1988119301.

17 Ibid., p. 205-206.

is Jean Brun insiste sur le fait intéressant que la vitesse, comme le temps. est complexe, bâtarde, issue de la conjonction de deux désirs hétérogènes, celui de gagner du temps et celui de dévorer de lespace; voir "ivresse et tristesse de la vitessce", Corps écrit, no 24 , décombre 1987, p. 33-43. 
104

perceptible que sur le fond de temps démultipliés: l'Amérique paraîtrait lente parce qu'il y règne un seul et unique tempo.

Que l'infarctus de Pierre survienne dans un avion n'est pas davantage surprenant. Sans doute faut-il voir là un écho à la peur qui a scandé l'histoire de la course technologique à la vitesse amorcée avec le $\mathrm{XIX}^{\mathrm{e}}$ siècle: dans L'invention de la vitesse, Studeny rappelle notamment les craintes que suscitait la vitesse des trains dans la première demie du $\mathrm{XIX}^{\mathrm{e}}$ siècle, vitesse pourtant point encore excessive (18 lieues à l'heure en 1844 !) ${ }^{19}$. Plus fondamentalement, l'avion est un multiplicateur de vitesse; sa vélocité s'additionne à celle de Pierre et provoque une rupture de l'équilibre des pressions externe et interne (pensons à la théorie de la pression formulée par le héros (p. 101) et à l'importance de la pressurisation dans la cabine des avions). Déjà, l'accélération de la vie américaine avait contribué à l'accélération du processus de vieillissement ${ }^{20}$; la vitesse de l'avion ne vient qu'achever ce processus.

En même temps que l'aéroplane représente un absolu de la vitesse, il en libère de façon paradoxale: chose curieuse, l'engin qui se déplace le plus rapidement paraît en fait ne pas bouger (c'est du moins le cas des avions de ligne). Voyager en avion, c'est non seulement nier l'espace, "biffer le monde " comme l'écrit Drieu la Rochelle, c'est aussi en quelque sorte se retirer du temps mesuré, tromper le temps cosmologique; cela est particulièrement sensible avec le décalage horaire, qui ajoute ou retranche des heures, fait se coucher et se lever le soleil dans un intervalle extrêmement bref, donne accès à une dimension inédite du temps, le contractant et le dilatant à l'envi. Juste avant de ressentir les premières atteintes de son mal, Pierre Nioxe note ceci:

Sans doute ne voyais-je l'univers sous son aspect tumultueux que parce que j'avais le nez dessus. On ne va vite qu'à ras du

19 "P. Andricux en 1844, prudent, propose un maximum de 18 lieues à l'heure, qui permettrait de traverser tout le territoire français en une journée et "parce que cette vitesse n'est pas nuisible: au-delà seulement commence la gêne atmosphérique et de la respiration" ": Studeny, op. cit., p. 223.

20) "Qu'il ne clépasse que de peu la trentaine, ce n'est pas croyable tant ses traits sont tirćs, tant sous le noir du chapeau la calotte des cheveux est déà blanchissante. Son nez est devenu un bec d'oiseau. Non seulement le visage n'a plus ses reflets jeunes et charmants d'autrefois, mais sa physionomic, c'est-itclire cette partic constante de notre figure sur laquelle s'appuient les diagnostics et les pronostics des médecins ou des bohémiennes; s'est régulièrement altérée penctant le voyagen (p. 253). 
sol. Dès que je prends du recul pour regarder ma vieille planète, elle me paraît morte. La vitesse, c'est un mot inventé par le ver de terre. (p. 256)

En plus de souligner à nouveau le caractère relatif de la vitesse, cette remarque introduit à une autre de ses dimensions fondamentales; la vitesse technologique moderne détache du sol, éloigne de ce que Studeny appelle le triple lieu familier: le pas, le jour, le lieu ${ }^{21}$. L'avion est l'emblème parfait de ce que Jean Chesneaux, dans De la modernité, a nommé le hors-sol ${ }^{22}$. C'est le cas non seulement parce l'avion permet d' "échapper à la terre" montrant du coup, et paradoxalement, qu'il n'y a "rien que la terre " 23 -, mais aussi parce qu'il établit un pur réseau totalement dégagé des accidents géographiques (et éventuellement politiques, historiques, etc.) de la planète. Le métro produit le même effet de hors-sol: il se déploie sans tenir compte du tracé des rues à la surface, de la cartographie officielle ou secrète de la ville; on pourrait dire la même chose du TGV et, plus généralement, des réseaux modernes de chemin de fer et d'autoroutes, qui d'ailleurs sont souvent représentés comme de purs tracés, sans référence aucune à la topographie.

L'bomme pressé manifeste exemplairement la dimension du hors-sol. Rappelons d'abord que Nioxe est antiquaire, et que cette profession se caractérise par l'échange de biens sans considération pour leur milieu culturel d'origine. Notons au surplus que le héros a participé à des fouilles archéologiques qui s'apparentent dans certains cas à un pillage ${ }^{21}$ : sous ce rapport, l'épisode de l'achat du cloître roman, de son démontage et de sa

21 Studeny, op. cit., p. 338. Studeny mentionne que Rilke, déjà en 1907, remarquait (que la vitesse fait tomber dans l'oubli le proche comme le lointain (p. 344-345).

22 Jean Chesneaux, De la modernité, Paris, la Découverte/Maspero, 1983, coll. "Cahiers libres".

23 "Fichapper à la terre" est le titre d'un chapitre de l'ouvrage de Studeny, Rien que la terre est celui d'un récit de voyage publié par Morand en 1926.

24 I In passage du récit, construit sur le contraste entre archéologie et technologie, fait particulièrement apercevoir la mise hors-sol clu patrimoine d'une civilisation disparue: Placide raconte que "Pierre, gagnant du terrain sur tout le monde. découvrait, tantôt dans des centres de fouilles, tantôt dans des collections privées, des objets plus vieux de dix ou quinze siècles que les trains d'acier ou les avions de cluralumin dans lesquels il les emportait, et comment cette "chasse au trésor" satisfaisait son besoin de "déplacements irationnels" $"(p .161)$. 
106

vente à la ville de Chicago se révèle particulièrement éloquent; y a-t-il, en effet, signe plus spectaculaire de la culture hors-sol que la reconstitution d'artefacts gigantesques à des milliers de kilomètres de leurs sites originaux? Il y a cependant beaucoup d'autres références au hors-sol disséminées dans le récit: le protagoniste n'a aucune attache sociale, n'appartient à aucun lieu, ne se fixe en aucun; il est pure trajectoire, pure ampleur; son corps même se récluit à une ligne, à un trait, à un profil ; son action est mouvement, son être est moment. Le hors-sol constitue en somme une forme d'abolition du monde dont la vitesse est un mode; au sens propre du terme, il est un non-lieu qui abolit le monde sensible.

Ces considérations conduisent à la théorie de Paul Virilio selon laquelle la vitesse accomplit la triple disparition du monde, des autres et de soi ${ }^{25}$. Je viens d'aborder la question de la suppression du monde; on a vu plus haut que la vélocité annihilait l'intimité, le rapport à l'autre; il s'agit maintenant de s'attacher au problème de l'effacement de soi par la vitesse. Pour Virilio, la vitesse nous rend absents à nous-mêmes; nous faisant passer de "l'être-là " au "n'être plus là ", elle nous amène à disparaître ${ }^{26}$ (on sait que Nioxe a le don de l'éclipse): "Le transport, écrit Rémy Paindavoine à la suite de Virilio, est un ravissement, une extase, un transport. Le passager, en toute rigueur, n'existe pas. Il passe. Il tré-passe. La vitesse implique un adieu à soi-même. 27 On relèvera, ici, la mention du trépas, qui trouve un troublant écho dans la mort du protagoniste de L'homme pressé; on mettra également cette citation en parallèle avec la confession étonnante de Nioxe: "Je n'existe pas, je préexiste; je suis un homme antidaté; non, je ne suis pas un homme, je suis un moment!" (p. 254). A peine anticipé, l'homme-moment - c'est dans sa nature - a déjà passé; son avenir est derrière lui: il a cessé d'exister, même comme souvenir.

Au terme du récit, Pierre Nioxe, tenu à l'immobilité à cause de son accident cardiaque, est irrémédiablement condamné à mourir, comme les requins qui s'asphyxient s'ils cessent de se mouvoir. En congé de la vitesse, il passe dans une autre vie, où c'est au tour des choses immobiles de se mettre à marcher

25 Voir à ce propos Rémy Paindavoine, op. cit., p. 620s.

26 Ibid., p. 621.

27 Ibicl, p. 622. 
(p. 45). Tout bien considéré, l'homme de la vitesse connaît le sort de toutes les technologies: sa destinée se solde logiquement par un bris du corps-machine, puisque, selon Virilio, toute technologie est porteuse d'un accident spécifique qui est l'envers et le prix clu progrès technique ${ }^{28}$. De sorte que L'bomme pressé paraît représenter une expérimentation romanesque de la capacité optimale de la machine humaine à générer et à supporter la vitesse.

28 Voir à ce sujet l'intéressante entrevue de François Ewald avec Paul Virilio, "Vitessce, guerre et vidéo", dans le Magazine littéraire, $\mathrm{n}^{\circ} 337$, novembre 1995. p. 96-103. 